FORMATION Formation emploi

Revue française de sciences sociales

137 | Janvier-Mars 2017

La formation continue en contexte : l'entreprise au cœur des enjeux

\title{
Edito : La formation continue en contexte
}

Jean-Frédéric Vergnies

\section{(2) OpenEdition}

Journals

Édition électronique

URL : http://journals.openedition.org/formationemploi/4968

DOI : 10.4000/formationemploi.4968

ISSN : 2107-0946

Éditeur

La Documentation française

Édition imprimée

Date de publication : 30 avril 2017

Pagination : 1-2

ISSN : 0759-6340

Référence électronique

Jean-Frédéric Vergnies, «Edito : La formation continue en contexte», Formation emploi [En ligne], 137| Janvier-Mars 2017, mis en ligne le 30 avril 2017, consulté le 30 octobre 2020. URL : http://

journals.openedition.org/formationemploi/4968; DOI : https://doi.org/10.4000/formationemploi.4968

(c) Tous droits réservés 


\title{
La formation continue en contexte
}

\author{
Jean-Frédéric Vergnies \\ Rédacteur en chef
}

De grandes régularités parcourent la formation continue, en France. Les grandes entreprises, certains secteurs forment davantage et cela bénéficie surtout aux cadres et aux plus diplômés. Les freins à la formation continue relèvent surtout de la charge de travail et du coût, mais aussi du niveau de formation initiale ou des contraintes familiales inégalement réparties selon le sexe ${ }^{1}$.

Ce dossier envisage la formation continue non seulement au niveau de l'individu ou de l'entreprise, mais aussi de manière plus contextuelle et systémique. Il invite à explorer les régulations de la formation continue, notamment au niveau de l'organisation de l'entreprise et des facteurs qui l'influencent. Il s'appuie sur des données usuelles comme le taux d'accès à la formation, la durée, ou le taux de participation financière des entreprises. En même temps, il propose d'aller plus loin en interrogeant, d'une part, les modes de régulation du financement de la formation continue et, d'autre part, l'organisation des entreprises, notamment en termes de gestion des ressources humaines et de politique de formation.

La formation continue s'inscrit ici dans un contexte et des interactions entre salariés, entreprises et institutions. De nouvelles générations d'enquêtes statistiques, dites couplées, contribuent à les analyser (Difes1 et 2 - dispositif d'information sur la formation employeur/salarié ; l'enquête Defis en cours - dispositif sur les formations et itinéraires des salariés) ${ }^{2}$.

Les deux premiers articles interrogent de manière qualitative les systèmes de régulation de la formation continue au niveau national. Isabelle Voirol-Rubido souligne les carac-

1 Quand la formation continue... Repères sur les pratiques de formation des employeurs et salariés, coordonné par Marion Lambert, Isabelle Marion-Vernoux, Céreq, avril 2014, 111 p : http://www.cereq.fr/publications/Ouvrages/Quand-la-formation-continue-Reperes-sur-les-pratiques-de-formation-des-employeurs-etsalaries.

2 http://www.cereq.fr/index.php/cereq/themes/Acces-aux-donnees-Themes/Enquetes-Formation-continue 
téristiques du système libéral suisse, en envisageant l'avenir possible de son financement auprès des partenaires sociaux. Josua Grabener, de son côté, compare la régulation du financement de la formation continue au niveau des branches, en France et en Italie, en pointant les proximités apparentes de structures. Pour l'auteur, les défis pour financer les formations longues certifiantes sont similaires dans les deux pays, mais en raison de mécanismes différents.

Comme le rappelle utilement Edward Lorenz dans sa postface, les systèmes de régulation de la formation professionnelle continue seraient plus efficaces s'ils prenaient en compte l'organisation du travail et les pratiques de gestion des ressources humaines, ce que s'emploient à explorer les autres articles du numéro. Ces travaux sont issus d'un séminaire piloté par le département formation et certification du Céreq.

Mathilde Guergoat-Larivière et Coralie Perez interrogent le lien entre conjoncture et formation continue. Il s'agit de mieux comprendre dans quelle mesure la formation continue peut relever plutôt de coûts à réduire ou plutôt d'investissements à optimiser.

Puis Josiane Vero et Jean-Claude Sigot montrent que certaines configurations organisationnelles, au-delà des grandes différences classiques de secteur, de taille..., sont plus favorables au développement d'une formation continue assumée par les salariés. Ils proposent aussi des classifications des politiques de formation des entreprises qui font ressortir que $43 \%$ des entreprises ne disposent pas encore de politique de formation formalisée.

Pour leur part, Ekaterina Melnik-Olive et Hélène Couprie s'intéressent plus précisément aux inégalités d'accès à la formation et d'accès à la promotion selon le sexe et les types de gestion des ressources humaines des entreprises. Le degré d'inégalités entre hommes et femmes en matière de formation continue et de promotion est fortement tributaire des pratiques de gestion des ressources humaines en termes de transparence de l'information, de réflexion stratégique, et de dialogue autour de la formation.

Enfin, Benoit Cart, Valérie Henguelle et Marie-Hélène Toutin interrogent aussi les liens entre formation continue et parcours professionnels. Ainsi, bénéficier d'une formation favorise-t-il le maintien en emploi dans l'entreprise ? Selon les auteurs, cela dépend du type de formation (générale ou spécifique), mais aussi du contexte, aussi bien au niveau de l'individu que de l'entreprise.

Bonne lecture. 